\title{
Fatores de risco para uso do tabaco em adolescentes de duas escolas do município de Santo André, São Paulo
}

\author{
Risk factors for tobacco use in adolescent students of two high schools of Santo André city, São Paulo, Brazil
}

\author{
Halley Ferraro Oliveira ${ }^{1}$, Lourdes Conceição Martins², Lígia de Fátima N. Reato ${ }^{3}$, Marco Akerman $^{4}$
}

\section{RESUMO}

Objetivo: Avaliar fatores de risco para uso de tabaco em estudantes de duas escolas do ensino médio do município de Santo André (SP) em 2005, bem como traçar o perfil do adolescente experimentador do fumo.

Métodos: Estudo transversal, com questionário padronizado, autoaplicável e anônimo aplicado a adolescentes escolares de ambos os sexos, matriculados no primeiro ano do ensino médio de duas escolas de Santo André, uma particular e outra pública. Foram levantadas as seguintes variáveis: idade, sexo, raça, prática de esportes, religião e hábitos do adolescente e da família. Definiu-se como "experimentador do fumo" o adolescente que experimentou cigarro (tabaco) e não continuou o hábito tabagista. Foi feita a análise descritiva com o teste do qui-quadrado e os fatores de risco para "experimentar fumo" foram analisados por regressão logística.

Resultados: Dos 232 questionários aplicados, $226(90 \%)$ foram respondidos, sendo 137 (61\%) na escola particular e $89(39 \%)$ na estadual. Dentre os entrevistados, 54 (24\%) referiram já ter experimentado tabaco uma vez na vida (35 na escola privada e 19 na pública). Foram fatores de risco significativos para experimentar fumo na escola particular: mãe fumante (OR 12,4; IC95\% 4,1-37,8) e amigos fumantes (OR 9,6; IC95\% 3,1-29,9). Na escola pública, os fatores de risco para a mesma variável foram: consumo de bebida alcoólica (OR 8,8; IC95\% 1,9-40,1), amigos fumantes, (OR 7,9 ; IC95\% 1,9-31,3) e contato com fumaça do cigarro (OR 6,4; IC95\% 1,6-26,2).

Conclusões: Observou-se influência significativa do meio ambiente do adolescente, inclusive social e familiar, para a experimentação do tabaco.
Palavras-chave: adolescente; comportamento do adolescente; tabagismo; tabaco.

\section{ABSTRACT}

Objective: To evaluate risk factors for tobacco use in high school students of two schools of the city of Santo Andre (São Paulo, Brazil) in 2005, as well as to describe the profile of the adolescent who uses tobacco.

Methods: Cross sectional study that applied a standardized anonimous self-answered questionnaire in adolescents of both genders registered in the first year of a public and a private high school of the city of Santo André (Brazil). The following variables were collected: age, gender, race, practice of sports, religion and habits of the family/adolescent. "Tobacco trier" was defined as those who tried cigarette and did not continue with smoking habits, excluding those who inhaled illicit drugs. Statistical analysis applied chi-square test and logistic regression.

Results: A total of $226(90 \%)$ questionnaires were answered: $137(61 \%)$ in the private and $89(39 \%)$ in the public school. Among the adolescents, 54 (24\%) used tobacco (35 - private school and 19\% - public school). Significant variables associated with tobacco use in the private school were: maternal smoking (OR 12.44; 95\% CI 4.10-37.79) and friends who are smokers (OR 9.58; 95\% CI 3.10-29.98). In the public school, the risk factors for smoking were: alcoholic beverage use (OR 8.78; 95\% CI 1.92-40.06), smoking among friends (OR 7.90; CI95\%: 1.99-31.29) and contact with cigarette smoke (OR 6.40; 95\% CI 1.56-26.22).

Endereço para correspondência:

Halley Ferraro Oliveira

Rod dos Naufragos, s/n - cond. Morada do Rio

Rua B, quadra G, lote 11A

CEP 49039-000 - Aracaju/SE

E-mail: halleyoliveira@yahoo.com.br

Conflito de interesse: nada a declarar

Recebido em: 25/3/09

Aprovado em: 26/9/09 
Conclusions: A significant influence of social and family environment was observed regarding the use of tobacco by adolescents.

Key-words: adolescent; adolescent behavior; smoking; tobacco.

\section{Introdução}

A adolescência é o período de transição entre a infância e a idade adulta caracterizado por intenso crescimento somático, desenvolvimento da capacidade de abstração e ampliação das referências sociais. Este período se manifesta através de transformações anatômicas, fisiológicas e mentais. É fortemente marcado pela puberdade e estabelecimento da capacidade reprodutiva. Nesta etapa da vida, o ambiente influencia a saúde e o desenvolvimento físico e psicossocial do adolescente ${ }^{(1)}$. Trata-se de uma época de experimentação natural, que pode levar ao aparecimento de comportamentos de risco, tais como: início precoce da atividade sexual, nãoutilização de proteção no ato sexual, hábitos alimentares inadequados, níveis baixos ou excessivos de atividade física, uso de tabaco, álcool e outras substâncias psicoativas. Desses, um dos comportamentos de risco mais difundidos na adolescência é o consumo experimental e recreativo de substâncias psicoativas, dentre elas o cigarro ${ }^{(2)}$.

O tabaco é a segunda droga mais consumida entre os jovens no mundo e no Brasil $^{(3,4)}$. Segundo a Organização Mundial de Saúde (OMS), o tabagismo é considerado um dos mais importantes problemas de Saúde Pública da atualidade. É a principal causa de morte evitável ${ }^{(3)}$ no mundo e mata mais pessoas do que a Síndrome da Imunodeficiência Adquirida (AIDS), os acidentes de trânsito, o alcoolismo, as drogas ilegais, os suicídios e homicídios juntos ${ }^{(3)}$. Desta forma, o tabagismo pode ser considerado uma pandemia, já que cerca de 5 milhões de indivíduos no mundo vão a óbito a cada ano por doenças relacionadas ao fumo ${ }^{(3)}$.

Sabe-se que $90 \%$ dos fumantes adultos começam a fumar antes dos 19 anos ( $75 \%$ entre 14 e 17 anos) e $50 \%$ dos experimentadores jovens se tornarão fumantes na idade adulta ${ }^{(5)}$. Portanto, é no período da adolescência média que o jovem inicia, desenvolve e consolida seu hábito tabagista, justamente na transição entre o ensino médio e o superior. Os fatores de risco conhecidos para o uso de tabaco na adolescência são: fumo entre amigos e irmãos, baixo desempenho acadêmico, idade mais velha durante a adolescência, gênero masculino, trabalho na adolescência, pais separados ${ }^{(6)}$ e curiosidade ${ }^{(7)}$.
Motivada pela importância do tema e escassez bibliográfica sobre o assunto na população adolescente, foi desenvolvida uma pesquisa em duas escolas da região do $\mathrm{ABC}$ paulista, inserida na Grande São Paulo. O objetivo foi identificar os fatores de risco associados ao uso do tabaco (experimentar ou continuar no hábito tabagista) em adolescentes de 14 a 18 anos, estudantes de duas escolas do ensino médio do município de Santo André (SP).

\section{Métodos}

O estudo foi transversal, com entrevistas por meio de questionário padronizado, autoaplicável e anônimo a adolescentes escolares de ambos os sexos, matriculados no primeiro ano do ensino médio (primeiro colegial) de duas escolas de Santo André, município da região metropolitana de São Paulo, no ano de 2005.

O protocolo consistiu em duas partes, totalizando 55 questões fechadas de múltipla escolha, algumas com espaço para preenchimento de lacunas. A primeira parte continha 37 perguntas, às quais todos responderam, e a segunda parte constava de 18 questões só para os fumantes. Foram levantadas as seguintes variáveis: idade, gênero, etnia (raça), prática de esportes, religião e hábitos do adolescente e da família quanto à utilização de drogas (lícitas e ilícitas). O questionário foi aplicado por entrevistadores selecionados e previamente treinados. O projeto foi aprovado pelo Comitê de Ética em Pesquisa da Faculdade de Medicina do ABC.

$\mathrm{Na}$ época do estudo, havia quase 30 mil estudantes do ensino médio em Santo André (escolas particulares e públicas). Desta forma, foram escolhidos aleatoriamente 300 alunos (amostra de 1\% sobre o total de alunos do ensino médio do município). Estudou-se uma amostra não-probabilística por conveniência, na qual todos os alunos que concordaram em participar e tiveram o termo de consentimento livre e esclarecido assinado pelos pais e/ou responsáveis foram incluídos.

Foram escolhidas duas escolas: uma da classe socioeconômica C e D (escola estadual e de periferia) e outra da classe socioeconômica A e B (escola particular da região central). As duas escolas tinham cinco classes do primeiro ano do ensino médio com 30 alunos em cada classe, perfazendo um total de 300 adolescentes. Todos os alunos foram convidados a participar da pesquisa. Foi aplicado o questionário a todos os presentes no momento da pesquisa, correspondendo a um total de 232 questionários aplicados. Considerando-se aqueles que não quiseram participar $(n=5)$ e os nulos $(n=1)$, 
este número foi reduzido para 226 questionários, sendo 137 (61\%) na escola particular e 89 (39\%) na pública.

A palavra "experimentador" foi utilizada para o adolescente que experimentou um ou, no máximo, dois cigarros nos últimos 30 dias e não persistiu no hábito tabagista. Foi considerado "fumante" o adolescente que fumou um ou mais cigarros nos últimos 30 dias e manteve o hábito tabagista.

As variáveis qualitativas foram descritas de acordo com a frequência de eventos e as quantitativas, por medidas de tendência central (média, mediana) e de dispersão (desvio padrão, valor máximo e valor mínimo). Para verificar a associação entre as variáveis qualitativas e o grupo de experimentadores ou não-experimentadores, foi aplicado o teste de qui-quadrado e o teste de comparação entre duas proporções. A análise de regressão logística univariada e múltipla foi utilizada para verificar, em cada escola, os fatores de risco para o adolescente experimentar ou não o tabaco ${ }^{(8)}$. O nível de significância adotado foi de $5 \%$ e utilizou-se o software SPSS 16.0 em todas as análises.

\section{Resultados}

A amostra analisada foi composta por 226 adolescentes, sendo $54(24 \%)$ respondidos por experimentadores e 172 (76\%) por não-experimentadores. Dos 54 experimentadores, $35(65 \%)$ eram procedentes da escola particular e 19 (35\%) da escola pública (teste de comparação entre duas proporções, $p<0,05)$.

A idade dos alunos de ambas as escolas variou de 14 a 18 anos, sendo a média de 14,8 anos. Um total de 137 participantes (61\%) eram do gênero feminino, observandose prevalência maior do gênero feminino também entre os experimentadores 38/54 (70\%). Em relação à raça, observou- se predominância brancos $(161-71 \%)$, seguida negros e pardos (40 - 18\%).

Quando questionados sobre a idade em que iniciaram a experimentação do tabaco, obteve-se a média de 12,1 anos na escola particular (desvio padrão $=2,6$ anos, variando entre quatro a 15 anos, e mediana de 13,1 anos) e uma média de 13,2 anos na escola pública (desvio padrão = 1,6 anos, variando entre nove e 15 anos, com mediana de 14 anos).

Quanto à reprovação, na escola particular houve 10 (19\%) reprovados entre os experimentadores versus 14 (8\%) entre os não-experimentadores, apontando para uma possível associação entre a experimentação do tabaco e o mau desempenho escolar. Em relação à escola pública, não se observou diferença significante.

Em relação ao item "não possuir religião", verificou-se diferença quando comparados os experimentadores com os não-experimentadores.

Quanto a fatores socioculturais, 26\% (14/54) dos experimentadores afirmaram não possuir nenhuma religião, assim como 18\% (31/172) dos não-experimentadores (Tabela 1). Ao considerar a prática esportiva como qualquer atividade física fora da escola, apenas $30 \%$ dos adolescentes (68/226) praticavam esportes, sem diferença entre os experimentadores e os não-experimentadores.

Dos entrevistados, $49(91 \%)$ experimentadores e $99(58 \%)$ não-experimentadores relataram ter consumido bebidas alcoólicas pelo menos uma vez na vida $(p<0,05)$. Quando questionados se conheciam alguém que usava drogas ilícitas, $44(81 \%)$ experimentadores de tabaco e 84 (49\%) não-experimentadores responderam afirmativamente. Com relação ao uso de drogas como maconha, ecstasy, crack e cocaína, 219 (97\%) dos entrevistados responderam que nunca usaram. Dos experimentadores de tabaco, 7 (13\%) responderam já

Tabela 1 - Distribuição da amostra por gênero, raça, reprovação escolar e religião

\begin{tabular}{|c|c|c|c|c|c|c|c|c|c|c|c|c|c|c|}
\hline \multirow{3}{*}{ Variáveis } & & \multicolumn{6}{|c|}{ Experimentadores $(n=54)$} & \multicolumn{6}{|c|}{ Não-experimentadores $(n=172)$} & \multirow{3}{*}{$\begin{array}{c}\text { Qui- } \\
\text { quadrado* }\end{array}$} \\
\hline & & \multicolumn{2}{|c|}{$\begin{array}{c}\text { Escola } \\
\text { particular }\end{array}$} & \multicolumn{2}{|c|}{$\begin{array}{l}\text { Escola } \\
\text { pública }\end{array}$} & \multicolumn{2}{|c|}{ Total } & \multicolumn{2}{|c|}{$\begin{array}{c}\text { Escola } \\
\text { particular }\end{array}$} & \multicolumn{2}{|c|}{$\begin{array}{l}\text { Escola } \\
\text { pública }\end{array}$} & \multicolumn{2}{|c|}{ Total } & \\
\hline & & $\mathbf{n}$ & $\%$ & $\mathrm{n}$ & $\%$ & $\mathrm{n}$ & $\%$ & $\mathbf{n}$ & $\%$ & $\mathbf{n}$ & $\%$ & $\mathbf{n}$ & $\%$ & \\
\hline Sexo & Feminino & 23 & 66 & 15 & 79 & 38 & 70 & 66 & 65 & 33 & 47 & 99 & 58 & $p=0,12$ \\
\hline & Branca & 28 & 52 & 11 & 20 & 39 & 72 & 81 & 47 & 41 & 24 & 122 & 71 & \\
\hline Raça & $\begin{array}{l}\text { Negra/ } \\
\text { parda }\end{array}$ & 2 & 4 & 6 & 11 & 8 & 15 & 9 & 5 & 23 & 13 & 32 & 19 & $p=0,81$ \\
\hline Reprovação & Sim & 10 & 29 & 1 & 5 & 11 & 20 & 14 & 14 & 3 & 4 & 17 & 10 & $p=0,11$ \\
\hline Religião & Não & 13 & 37 & 1 & 5 & 14 & 26 & 21 & 21 & 10 & 14 & 31 & 18 & $p=0,44$ \\
\hline
\end{tabular}

Reprovação: reprovação na escola; *total dos experimentadores versus total dos não-experimentadores. 
ter usado drogas ilícitas pelo menos uma vez na vida versus nenhum dos não-experimentadores (Tabela 2).

Com relação ao fato de ter pai fumante e de os cinco melhores amigos fumarem, observou-se uma associação significativa entre essas variáveis e os grupos de estudo $(p<0,05)$. Quanto à presença de pai fumante, 43\% (23/54) dos experimentadores e 21\% (36/172) dos não-experimentadores responderam afirmativamente. Em relação às mães, a ausência de tabagismo foi maior entre não-experimentadores (96/172, 56\%), quando comparados aos experimentadores $(25 / 54,46 \%)$. Irmãos mais velhos fumantes foram relatados por 19\% (10/54) dos experimentadores e 9\% (15/172) dos não-experimentadores. Já com relação aos amigos, $70 \%$ (38/54) dos experimentadores relataram que possuíam amigos fumantes em comparação a 28\% (49/172) dos nãoexperimentadores (Tabela 3).

A Tabela 4 apresenta a análise de regressão logística univariada para cada uma das escolas (pública e particular). Na escola pública, verificou-se que as seguintes variáveis podem ser consideradas fatores de risco para o aluno experimentar a droga: sexo feminino, ingestão de bebida alcoólica, drogas ilícitas, amigos usuários. Já na escola particular foram identificados como fatores de risco: reprovação, bebida alcoólica, drogas ilícitas, pai fumante, mãe fumante, amigos fumantes e namorado fumante.

A Tabela 5 apresenta a análise da regressão logística múltipla. Nesse modelo, as variáveis consideradas fatores de risco para o aluno experimentar tabaco foram: mãe e amigos fumantes na escola particular e bebida alcoólica e amigos fumantes na escola pública.

\section{Discussão}

No estudo em questão, houve maior número de experimentadores na escola particular do que na pública. Neste aspecto, observou-se divergência em relação à literatura pesquisada, pois alguns autores relatam que nas escolas públicas há mais fumantes, enquanto outros relatam que nas escolas particulares este número é maior ${ }^{(9-12)}$.

Entre os experimentadores, há prevalência do gênero feminino, o que coincide com o levantamento do Centro Brasileiro de Informações sobre Drogas Psicotrópicas (CEBRID $)^{(13)}$, embora, em trabalho realizado na América do Sul por Malcon et $a l^{(6)}$, não tenha sido encontrada diferença relacionada ao gênero. Em estudo realizado entre 2002 e 2003 com escolares de 12 capitais brasileiras, foi encontrada uma prevalência de experimentação entre 36 e 58\% no gênero masculino e 31 e $55 \%$ no gênero feminino ${ }^{(7)}$. Entretanto, vale salientar que, conforme Pinto ${ }^{(11)}$, as meninas estão usando o tabaco cada vez mais e as mulheres têm apresentado taxa de experimentação e hábitos tabagistas iguais ou maiores do que os homens.

A idade de início do hábito tabagista atualmente ocorre com 12,8 anos $^{(13,14)}$. Quanto mais velho o adolescente se torna, mais experimenta ou continua a fumar ${ }^{(15,16)}$. Segundo Nascimento ${ }^{(14)}$, os adolescentes entram em contato com o tabagismo muito cedo, principalmente na fase de adolescência média, período em que há um maior risco, independentemente do gênero e do grau de escolaridade dos pais. Em outro estudo, Menezes ${ }^{(12)}$ relata que meninos, filhos de mães solteiras e de pais com baixa escolaridade, têm maior probabilidade de fumar na adolescência. Meninas pertencentes a famílias de baixa renda, com mães que fumaram durante a gravidez e pais com problemas relacionados ao álcool, apresentam maior probabilidade de fumar ${ }^{(12)}$.

Fraga $^{(17)}$ relata que adolescentes fumantes praticam menos exercício físico e, em torno de $35 \%$, começam a fumar no mesmo ano em que deixam de praticar esporte. Estudo francês realizado na cidade de Lorraine, em 2004, encontrou $34 \%$ de atletas fumantes ${ }^{(18)}$. Embora Arvers ${ }^{(19)}$ relate que a atividade esportiva moderada (uma a oito horas por semana) é fator protetor para o uso de cigarro e maconha, a prática do esporte de forma intensa (mais de oito horas por semana) pode ser fator de risco para o uso de outras drogas ilícitas. No presente estudo, não houve diferenças entre experimentadores e não-experimentadores quanto à prática esportiva, mas o tamanho da amostra limita a possibilidade de generalizar esse resultado.

Quanto à religião, o resultado encontrado foi compatível com os trabalhos de Dalgalarrondo e Wallace ${ }^{(20,21)}$, que mostram uma relação positiva entre religião/raça e proteção de adolescentes quanto ao uso de substâncias psicoativas, embora outros autores relatem que a experimentação de tabaco e maconha não apresenta associação com a religiosidade ${ }^{(22)}$.

No que se refere ao consumo de bebidas alcoólicas, dentre aqueles que informaram ter bebido, a maioria era experimentadora de tabaco, delineando uma associação positiva entre uso de bebida alcoólica e experimentação do fumo. Este resultado foi semelhante ao de outros estudos que reforçam a relação entre álcool e tabagismo ${ }^{(23-28)}$.

Em relação às drogas ilícitas, parece haver associação positiva com a experimentação do fumo. A literatura indica que estudantes que utilizam o cigarro também são usuários de outras drogas ${ }^{(19,21,22,27,28)}$ e, segundo o CEBRID ${ }^{(13)}$, existe 

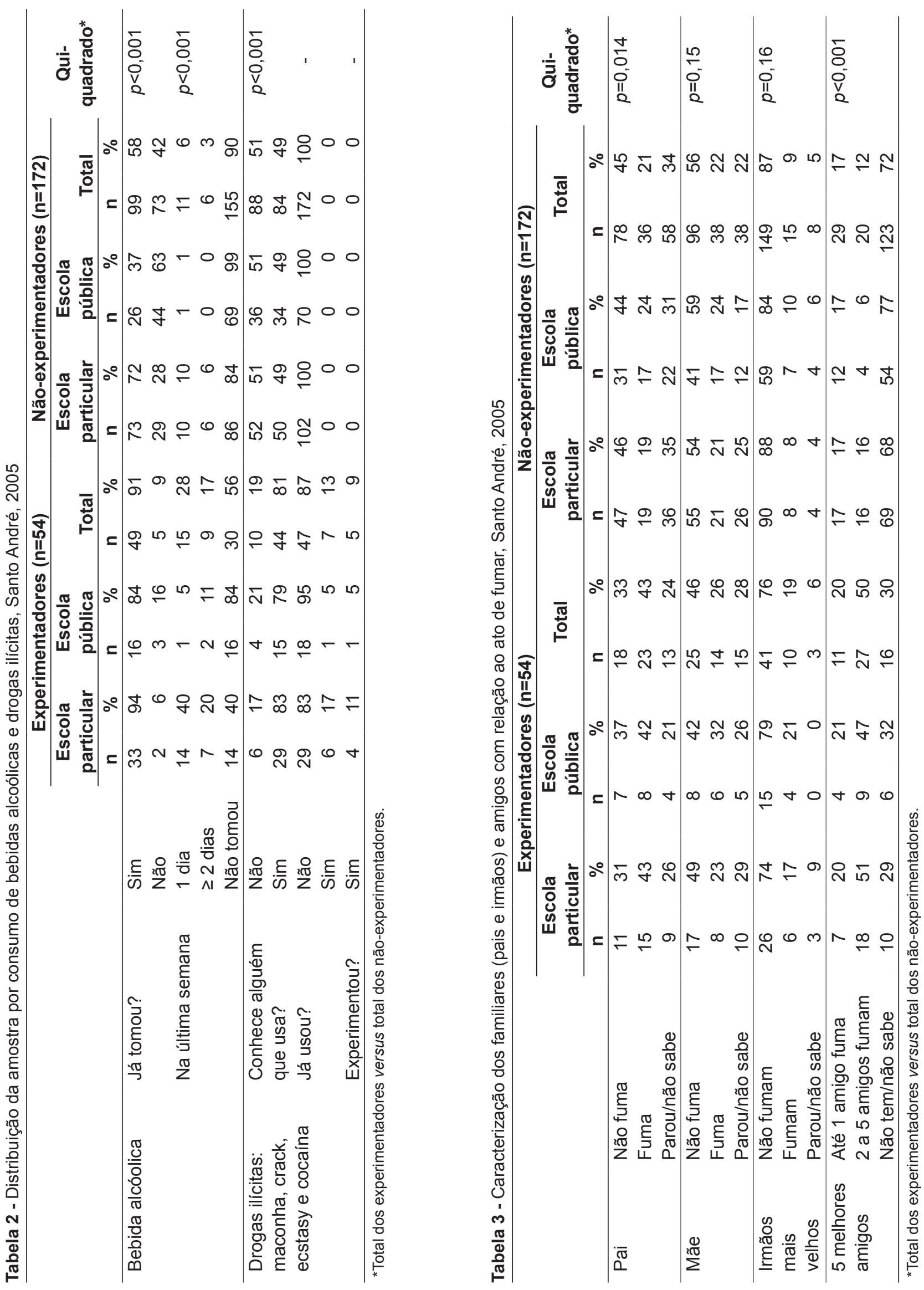
Tabela 4 - Associação entre uso de tabaco e fatores de risco em adolescentes escolares

\begin{tabular}{|c|c|c|c|c|}
\hline & \multicolumn{2}{|c|}{ Escola particular } & \multicolumn{2}{|c|}{ Escola pública } \\
\hline & OR & IC95\% & OR & IC95\% \\
\hline \multicolumn{5}{|l|}{ Sexo } \\
\hline Feminino & 1,05 & $0,47-2,34$ & 4,21 & $1,27-13,94$ \\
\hline \multicolumn{5}{|l|}{ Raça } \\
\hline Negra & 0,64 & $0,31-3,16$ & 0,97 & $0,32-2,97$ \\
\hline Amarela & 0,48 & $0,06-4,18$ & 1,24 & $0,22-7,03$ \\
\hline Não sabe & 1,93 & $0,51-7,34$ & - & - \\
\hline \multicolumn{5}{|l|}{ Reprovação } \\
\hline \multicolumn{5}{|l|}{ Religião } \\
\hline Não & 1 & - & 1 & - \\
\hline Sim & 0,44 & $0,19-1,01$ & 3 & $0,36-25,04$ \\
\hline \multicolumn{5}{|l|}{ Esporte } \\
\hline Não & 1 & - & 1 & - \\
\hline Sim & 0,84 & $0,36-1,94$ & 0,24 & $0,05-1,13$ \\
\hline \multicolumn{5}{|c|}{ Bebida alcoólica } \\
\hline Não & 1 & - & 1 & - \\
\hline Sim & 6,56 & $1,48-29,11$ & 9,03 & $2,40-33,96$ \\
\hline \multicolumn{5}{|c|}{ Drogas ilícitas } \\
\hline Não & 1 & - & 1 & - \\
\hline Sim & 5,03 & $1,92-13,14$ & 3,97 & $1,20-13,16$ \\
\hline \multicolumn{5}{|l|}{ Pai fuma } \\
\hline Não & 1 & - & 1 & - \\
\hline Sim & 3,12 & $1,34-7,25$ & 2,05 & $0,71-5,96$ \\
\hline \multicolumn{5}{|l|}{ Mãe fuma } \\
\hline Não & 1 & - & 1 & - \\
\hline Sim & 10,56 & $4,16-26,86$ & 1,28 & $0,42-3,89$ \\
\hline \multicolumn{5}{|c|}{ Irmãos fumam } \\
\hline Não & 1 & - & 1 & - \\
\hline Sim & 2,65 & $0,85-8,34$ & 2,29 & $0,59-8,84$ \\
\hline \multicolumn{5}{|c|}{ Amigos fumam } \\
\hline Não & 1 & - & 1 & - \\
\hline Sim & 6,6 & $2,58-16,88$ & 6,37 & $2,07-19,53$ \\
\hline \multicolumn{5}{|c|}{ Namorado fuma } \\
\hline Não & 1 & - & 1 & - \\
\hline Sim & 12,39 & $1,33-115,01$ & - & - \\
\hline
\end{tabular}

OR: Odds Ratio; IC95\%: intervalo de confiança de 95\%. 
Tabela 5 - Associação entre uso de tabaco e fatores de risco em adolescentes escolares

\begin{tabular}{lcccc}
\hline & \multicolumn{2}{c}{ Escola particular } & \multicolumn{2}{c}{ Escola pública } \\
\cline { 2 - 5 } & OR & IC95\% & OR & IC95\% \\
\hline Bebida alcoólica & - & - & 1 & - \\
Não & - & - & 8,78 & $1,92-40,06$ \\
Sim & & - & - & - \\
Mãe fuma & 1 & $4,10-37,79$ & - & - \\
Não & 12,44 & - & 1 & - \\
Sim & 1 & - & 7,9 & $1,99-31,29$ \\
Amigos & 9,58 & $3,10-29,98$ & & \\
Não &
\end{tabular}

OR: Odds Ratio; IC95\%: intervalo de confiança de 95\%.

uma forte associação entre o uso de substâncias psicotrópicas e o rendimento escolar.

A influência dos pais e irmãos mais velhos bem como dos cinco melhores amigos como fator de risco para a experimentação do fumo coincide com dados encontrados na literatura $^{(16,17)}$. Vários autores ${ }^{(6,7,14,19,29)}$ citam como fator de risco para o tabagismo o fato de os adolescentes possuírem amigos fumantes. De acordo com a literatura ${ }^{(6,15,17,30)}$, dentre os aspectos que influenciam o início do hábito do tabagismo, destacam-se: gênero, idade, nível socioeconômico, pais, irmãos ou amigos fumantes, rendimento escolar (não estudar ou ser repetente), esporte (não praticar), bebidas alcoólicas no último mês, trabalho remunerado exercido pelo adolescente e separação dos pais. Destes, considera-se como principal fator associado ao uso do tabaco o hábito de fumar entre irmãos e amigos ${ }^{(6,30)}$.

Assim sendo, os resultados encontrados no presente estudo foram compatíveis com a literatura, identificando-se como fatores de risco para experimentação de fumo entre adolescentes na escola pública: gênero feminino, ingestão de bebida alcoólica, drogas ilícitas e amigos fumantes; e na escola particular: reprovação escolar, consumo de bebida alcoólica, drogas ilícitas, pai, mãe, amigos e namoradas(os) fumantes.

Finalmente, embora se trate de estudo local, a pesquisa pode contribuir para traçar uma estratégia de intervenção frente à utilização de tabaco por adolescentes. Sugere-se que o cenário adotado seja o mesmo do campo de pesquisa: as escolas, espaços tradicionais de formação, modelo e, consequentemente, de prevenção e promoção da saúde.

\section{Agradecimentos}

À Liga de Hebiatria da FMABC, aos diretores das escolas analisadas, aos adolescentes que aceitaram participar e a todos que contribuíram de forma direta ou indireta para a realização deste trabalho.

\section{Referências bibliográficas}

1. Crespin J. Breve história da medicina da adolescência. In: Crespin J, Reato LFN, editors. Hebiatria: medicina da adolescência. São Paulo: Rocca; 2007. p. 4-9.

2. Epps RP, Manley MW, Glynn TJ. Tobacco use among adolescents. Strategies for prevention. Pediatr Clin North Am. 1995;42:389-402.

3. World Health Organization [homepage on the Internet]. Tobacco free initiative (TFI): why is tobacco a public health priority? [cited 2010 Apr 28]. Available from: http://www.who.int/tobacco/health_priority/en

4. Organização Pan-Americana de Saúde [homepage on Internet]. Tabaco nas Américas [cited 2010 Apr 28]. Available from: http://www.opas.org.br/sistema/ fotos/tabaco.htm

5. Goldfarb LC. Tabagismo: estudo em adolescentes e jovens. In: Schor N, Mota MS, Branco VC, editores. Cadernos juventude, saúde e desenvolvimento. Brasília: Ministério da Saúde; 1999. p. 162-72.

6. Malcon MC, Menezes AM, Maia MF, Chatkin M, Victora CG. Prevalência

e fatores de risco para tabagismo em adolescentes na América do Sul: uma revisão sistemática da literatura. Rev Panam Salud Publica 2003;13: 222-8.

7. Instituto Nacional do Câncer [homepage on the Internet]. VIGESCOLA Vigilância de tabagismo em escolares. Dados e fatos de 12 capitais brasileiras [cited 2010 Apr 28]. Available from: http://www.inca.gov.br/vigescola/docs/ vigescola_completo.pdf

8. Kleinbaum DG, Kupper LL, Muller KE, Nizam A. Applied regression analysis and other multivariable methods. $3^{\text {rd }}$ ed. Belmont: Duxbury Press; 1998.

9. Iglesias V, Cavada G, Silva C, Cáceres D. Early tobacco and alcohol consumption as modifying risk factors on marijuana use. Rev Saude Publica 2007;41:517-22.

10. Bolzán A, Peleiro R. Smoking during early adolescence. A study in schoolchildren from Argentina. J Pediatr (Rio J) 2003;79:461-6. 
11. Pinto DS, Ribeiro SA. Variáveis relacionadas à iniciação do tabagismo entre estudantes do ensino médio de escola pública e particular na cidade de Belém - PA. J Bras Pneumol 2007;33:558-64.

12. Menezes AM, Hallal PC, Horta BL. Early determinants of smoking in adolescence: a prospective birth cohort study. Cad Saude Publica 2007;23:347-54.

13. Galduróz JC, Noto AR, Fonseca AM, Carlini EA. V Levantamento nacional sobre o consumo de drogas psicotrópicas entre estudantes do ensino fundamental e médio da rede pública de ensino nas 27 capitais brasileiras 2004. São Paulo: Centro Brasileiro de Informações sobre Drogas Psicotrópicas (CEBRID), Departamento de Psicobiologia da Escola Paulista de Medicina; 2004.

14. Nascimento D, Soares EA, Feitosa S, Colares V. Tobacco smoking habit among adolescents in the city of Recife and associated factors. Rev Odonto Cienc 2005;20:348-53.

15. Horta BL, Calheiros P, Pinheiro RT, Tomasi E, Amaral KC. Tobacco smoking among teenagers in an urban area in Southern Brazil. Rev Saude Publica 2001;35:159-64.

16. Machado Neto AS, Cruz AA. Smoking among school adolescents in Salvador (BA). J Pneumol 2003;29:264-72.

17. Fraga S, Ramos E, Barros H. Smoking and its associated factors in Portuguese adolescent students. Rev Saude Publica 2006;40:620-6.

18. Laure $P$, Lecerf $T$, Friser A, Binsinger C. Drugs, recreational drug use and attitudes towards doping of high school athletes. Int J Sports Med 2004;25:133-8.

19. Arvers $P$, Choquet M. Sporting activities and psychoactive substance use. Data abstracted from the French part of the European School Survey on Alcohol and other Drugs (ESPAD 99). Ann Med Interne 2003;154:S25-34.

20. Dalgalarrondo P, Soldera MA, Corrêa Filho HR, Silva CA. Religião e uso de drogas por adolescentes. Rev Bras Psiquiatr 2004;26:82-90.
21. Wallace JM Jr, Brown TN, Bachman JG, LaVeist TA. The influence of race and religion on abstinence from alcohol, cigarettes and marijuana among adolescents. J Stud Alcohol 2003;64:843-8.

22. Chen CY, Dormitzer CM, Bejarano J, Anthony JC. Religiosity and the earliest stages of adolescent drug involvement in seven countries of Latin America. Am J Epidemiol 2004;159:1180-8.

23. Newman K, Harrison L, Dashiff C, Davis S. Relationships between parenting styles and risk behaviors in adolescent health: an integrative literature review. Rev Latin Am Enfermagem 2008;16:142-50.

24. Andretta I, Oliveira MS. A study of the effects of motivational interviewing on adolescent offenders. Estud Psicol 2008;25:45-53.

25. Iglesias V, Cavada G, Silva C, Cáceres D. Consumo precoz de tabaco y alcohol como factores modificadores del riesgo de uso de marihuana. Rev Saude Publica 2007;41:517-22.

26. Horta RL, Horta BL, Pinheiro RT, Morales B, Strey MN. Tobacco, alcohol, and drug use by teenagers in Pelotas, Rio Grande do Sul State, Brazil: a gender approach. Cad Saude Publica 2007;23:775-83.

27. Everett SA, Malarcher AM, Sharp DJ, Husten CG, Giovino GA. Relationship between cigarette, smokeless tobacco, and cigar use, and other health risk behaviors among US High School Students. J Sch Health 2000;70:234-40.

28. Beck F, Legleye S, Spilka S. Polyconsommation de substances psychoactives (alcool, tabac et cannabis) dans la population générale française en 2005. Press Med 2008;37:207-15.

29. Malcon MC, Menezes AM, Chatkin M. Prevalence and risk factors for smoking among adolescents. Rev Saude Publica 2003;37:1-7.

30. Ministério da Saúde e Instituto Nacional do Câncer (INCA) [homepage on the Internet]. Jovem, mulher e tabaco [cited 2010 Apr 28]. Available from: http:// www.inca.gov.br/tabagismo/frameset.asp?item=jovem\&link=namira.htm 